\section{Urinary dysfunction in women with multiple sclerosis: analysis of 61 patients from Rio de Janeiro, Brazil}

\author{
Camila Rodrigues de Almeida, ${ }^{1}$ \\ Kennedy Carneiro, ${ }^{1}$ Rossano Fiorelli, ${ }^{1}$ \\ Marco Orsini, ${ }^{2}$ \\ Regina Maria Papais Alvarenga ${ }^{1}$ \\ ${ }^{1}$ Neurology Department, Center of \\ Biological and Health Sciences, State \\ University of Rio de Janeiro (UNIRIO); \\ ${ }^{2}$ Science Rehabilitation, University Center \\ Augusto Motta (UNISUAM), Rio de \\ Janeiro, Brazil
}

\section{Abstract}

The aim of the present study is to describe the type and frequency of bladder dysfunction in a series of female patients with multiple sclerosis (MS) from Rio de Janeiro, and analyze the role of the urologist in the multidisciplinary team. A team of urologists and a neurologist from the Hospital da Lagoa, Rio de Janeiro, Brazil, interviewed female patients with MS about illness onset, urologic follow-up since diagnosis, current stage of the disease and current urological symptoms. The interview was followed by an urodynamic testing, ultrasound of the urinary tract and urinalysis. Data resulting from the interviews and exam results were gathered and submitted to statistical evaluation. Sixty one patients were evaluated, with average age of 41.4 years. Urinary symptoms such as urinary incontinence, urinary hesitancy, urinary retention, urinary urgency and incomplete bladder emptying were reported in $44 \%$ of patients as initial signs of MS disease. Mean disease duration was 8 years and all patients (100\%) with the primary progressive form of the disease and $63.5 \%$ with the relapsingremitting presentation had urological symptoms. Analysis of complementary exams showed that $37.7 \%$ of urinalysis, $8.2 \%$ of the urinary tract ultrasound exams and $66.7 \%$ of the urodynamic evaluations were abnormal and the most frequent abnormality were overactive neurogenic bladder. Only 4 patients (6.6\%) had seen an urologist during the course of their disease and only $1.6 \%$ had performed an urodynamic evaluation. This study shows a high prevalence (68\%) of urinary dysfunction in a female population with MS. Urologic care should be part of the multidisciplinary team since the beginning of the disease. An urodynamic evaluation and simple urinalysis should be included in the routine testing during disease follow-up due to the high incidence of neurogenic bladder and other urologic complications.

\section{Introduction}

Multiple Sclerosis (MS) is an idiopathic inflammatory-demyelinating disease of the central nervous system. International studies have shown that patients can present several forms of lower urinary tract dysfunction, which are referred in general as Neurogenic Bladder. ${ }^{1}$ Patients commonly experience symptoms such as increased urinary frequency, urinary urgency and urgency urinary incontinence (UUI). Urodynamic evaluation usually shows a predominance of detrusor overactivity. ${ }^{2-4}$ Voiding dysfunction is a common finding, occurring in $75 \%$ to $90 \%$ of the cases..$^{2,3}$ Bladder involuntary contractions often occur simultaneously to varying degrees of contraction of the external urinary sphincter, with consequent sphincter asynchrony that can be clearly recognized in video urodynamics. Low complacency, urinary residue after voiding, sphincter dyssynergy and high voiding pressure are factors that worsen the prognosis of neurological patients and may result in hydronephrosis, urinary tract infections and kidney failure.5-8 Regular urological evaluations are thus recommended, as $17.5 \%$ of the patients are eventually affected in the high urinary tract to a certain degree.., 10

MS is considered the most common neurological disease affecting young Caucasian individuals in the Northern Hemisphere. Brazil, however, has a warm climate and a mixed ethnic background, with a low prevalence of the disease. 11 Publications about the natural history of MS dated 1990 (South Atlantic Project). ${ }^{11}$ In a multicenter study on the profile and clinical course of MS in Brazil, 11 the long-term neurological dysfunction in MS was studied in 522 patients from 22 neurological centers, $90 \%$ with relapsing remitting clinical course (RRMS) and 10\% with primary progressive disease (PPMS)..$^{12-15}$ The bladder and bowel functional system was the third most affected system, with motor dysfunction being the most common disability, causing significant gait impairment.

In this study, we analyzed urinary symptoms and the results of complementary exams in female patients with MS in the city of Rio de Janeiro, Brazil. This is the first national study on this subject.

\section{Materials and Methods}

Our team interviewed 70 patients with idiopathic inflammatory demyelinating disease in the referenced MS neurological clinics at Hospital da Lagoa in Rio de Janeiro, Brazil. The study was approved by the Ethics Committee of Gaffree e Guinle University
Correspondence: Camila Rodrigues de Almeida Rua Republica do Peru, 193, 701, Copacabana, 22021040, Rio de Janeiro, Brazil.

E-mail: fiorellicamila@hotmail.com

Key words: multiple sclerosis, urinary dysfunction, urodynamics.

Contributions: the authors contributed equally.

Conflict of interests: the authors declare no potential conflict of interests.

Received for publication: 21 June 2012.

Revision received: 29 July 2013.

Accepted for publication: 5 September 2013.

This work is licensed under a Creative Commons Attribution NonCommercial 3.0 License (CC BYNC 3.0).

(C) Copyright C. Rodrigues de Almeida et al., 2013 Licensee PAGEPress, Italy

Neurology International 2013; 5:e23

doi:10.4081/ni.2013.e23

Hospital - HUGG. The study included only female patients who met the McDonald criteria (2001) for MS from the South Atlantic project. ${ }^{16}$ Patients were selected from the reference center for MS at Hospital da Lagoa, in Rio de Janeiro, Brazil. Exclusion criteria were male gender (because of anatomic factors with the prostate) and diagnosis of other demyelinating disease other than MS. All patients were interviewed and data referring to duration of disease, characterization of neurologic and urologic symptoms, concomitant urologic evaluation and complementary exams were collected. All initial and current symptoms were assessed.

After the interview, complementary investigation including urinalysis, ultrasound of the urinary and tract urodynamic testing were ordered. Patients were classified according to the Kurtzke Expanded Disability Status Scale (EDSS). ${ }^{13}$ The study design was bidirectional. At the first interview, an anamnesis was carried out focusing on neurological manifestations of the disease at onset, urological history and current urinary complaints. The follow-up protocol consisted of urologic complementary exams, such as urodynamic evaluation, ultrasonography of the urinary tract, examination of evidence of abnormal urinary sediment in simple urinalysis, and subsequent visits to review the results and offer specific treatment.

Current urinary symptoms were classified according to Kurtzke's functional system scale, FS: (Functional System) bladder and bowel adapted for the evaluation of bladder dysfunction, 14,17 and is described as follows: 0: Normal, 1: Mild urgency or urinary retention, 2: Moderate urinary dysfuntion (urethral selfintermittent catheterization or manual com- 
pression to empty the bladder), 3: Frequent urinary incontinence, 4: Need for almost constant bladder catheterization, 5: Loss of bladder function.

\section{Statistical analysis}

For statistical evaluation, we used the chisquare to compare discrete variables, such as urological abnormalities and clinical presentation. The McNemar chi-square test was used to compare discrete variables at different moments of the disease (urological abnormalities at the beginning of disease and at the moment of the interview). Finally, the MannWhitney test was used to compare two variables, one discrete and the other ordinal or numerical and to calculate the confidence interval to estimate the study inaccuracy. Spearman's rank correlation was performed and classified as: very weak ( 0.0 to 0.19$)$, a weak ( 0.20 to 0.39$)$, moderate ( 0.40 to 0.59 ), strong ( 0.60 to 0.79$)$ or a very strong correlation ( 0.80 to 1.00$)$; P-values $<0.05$ were considered statistically significant.

\section{Results}

According to the aforementioned criteria, 61 patients were selected and 9 patients were excluded (5 man and 4 with other Idiopathic inflammatory demyelinating diseases IIDD). Of those, 52 (85.2\%) had RRMA and 9 (14.8\%) had PPMS. The population studied had a mean age of 41.4 years (standard deviation: 10.9). The mean age at disease onset was 33.4 years and the mean disease duration was 8 years ( 6 months to $27 \mathrm{yrs}$ ).

\section{Neurological manifestations of multiple sclerosis at onset of disease}

MS presented a clinically isolated syndrome in 18 (29.5\%) patients, characterized by muscle weakness in 1 or more limbs $(n=8)$, visual impairment $(n=3)$, gait impairment $(n=1)$ and association of paresis with numbness in one or more limbs $(n=6)$. Urinary symptoms at onset, such as urinary incontinence, urinary hesitancy, urinary retention, urinary urgency and incomplete emptying of the bladder, were reported by $27(44.2 \%)$ patients and were always associated with other neurological symptoms, suggesting that different areas in the central nervous system were affected, as shown in Figure 1. In the remaining 16 patients, MS had already started with a multifocal pattern, affecting different functional systems, yet sparing the bladder control system. Overall, motor dysfunction was the most frequent initial manifestation of the disease, followed by bladder dysfunction.

\section{Long-term urologic manifestations in multiple sclerosis}

Interview was held after a mean duration of disease of 8 years (0.7). Urological symptoms were reported by 42 patients (68\%) along with

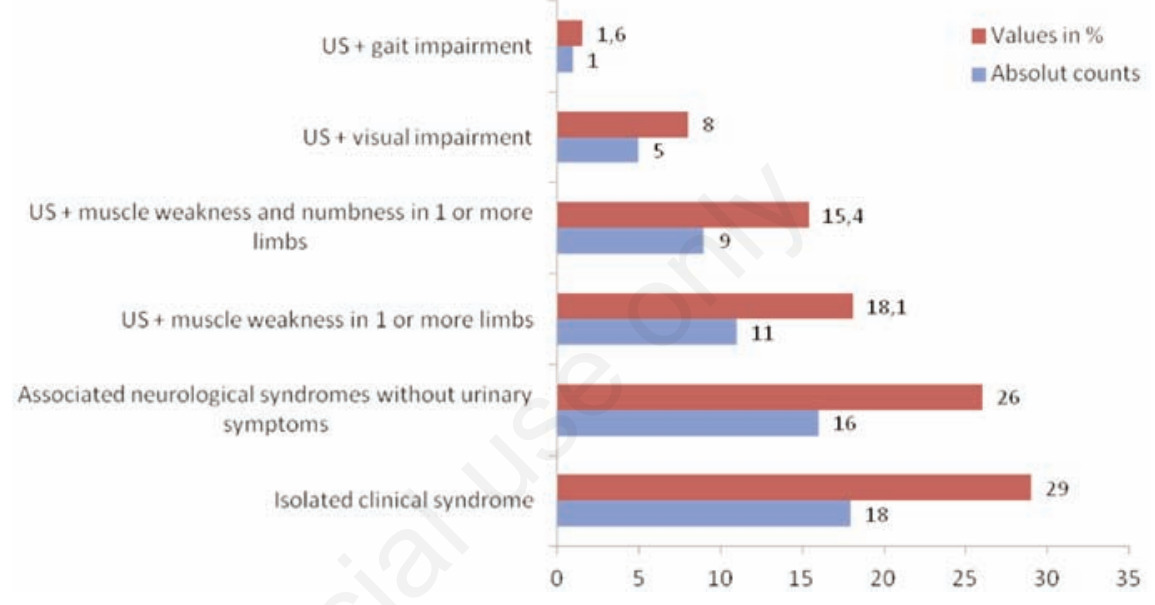

Figure 1. Multiple sclerosis initial neurological manifestations (n=61).

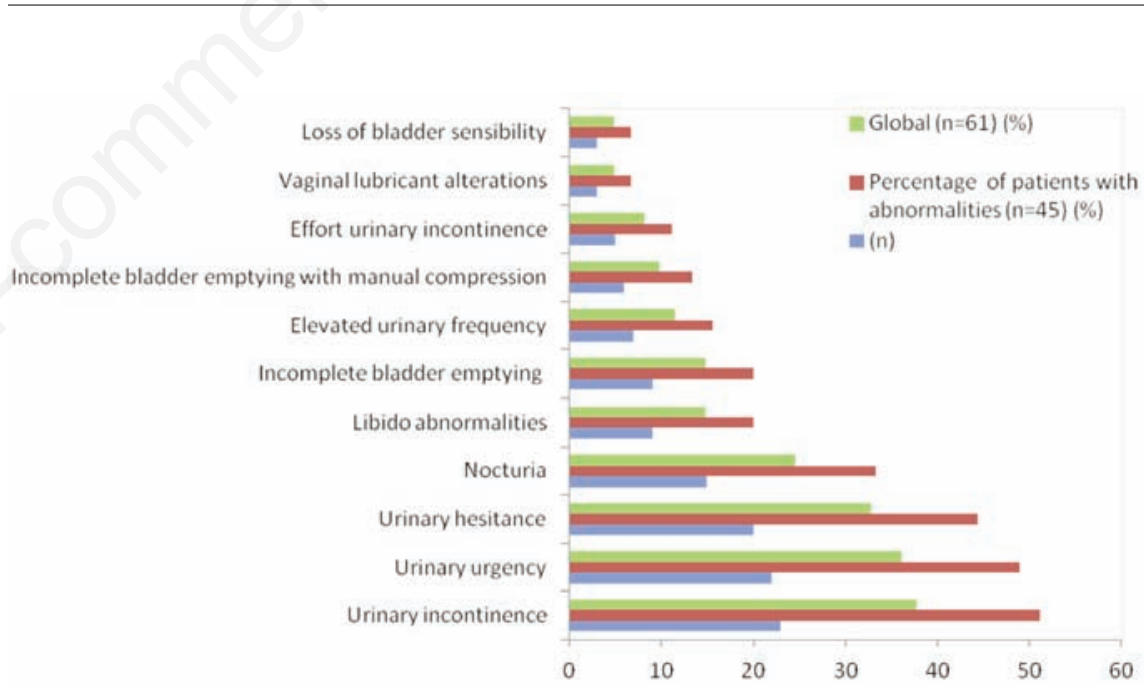

Figure 2. Urological abnormalities in multiple sclerosis patients $(n=61)$.

Table 1. Urological abnormalities in multiple sclerosis patients according to clinical presentation.

\begin{tabular}{|c|c|c|c|c|c|c|}
\hline \multirow[t]{3}{*}{ Multiple sclerosis clinical presentation } & \multirow{2}{*}{\multicolumn{2}{|c|}{ Total }} & \multicolumn{4}{|c|}{ Urological abnormalities } \\
\hline & & & \multicolumn{2}{|c|}{ Yes } & \multicolumn{2}{|c|}{ No } \\
\hline & n. & $\%$ & n. & $\%$ & n. & $\%$ \\
\hline Relapsing-remitting (MS-RR) & 52 & 100 & 33 & 63.5 & 19 & 36.5 \\
\hline Primary progressive (MS-PP) & 9 & 100 & 9 & 100 & 0 & 0.0 \\
\hline Total & 61 & 100 & 42 & 68.9 & 19 & 31.1 \\
\hline
\end{tabular}


other neurologic manifestations of MS.

The relative and absolute frequencies of these symptoms are summarized in Figure 2. Urinary incontinence was the most prevalent symptom, followed by urinary urgency and urinary hesitation. A comparison of the prevalence of urinary disorders in different clinical presentations of MS is summarized in Table 1.

\section{Classification of urinary dysfunction}

Applying Kurtzke's Functional System Scale for bladder function, we observed that at the time of the interview, $31.1 \%$ of patients were asymptomatic from an urological standpoint, whereas $68.9 \%$ related some kind of change in bladder control, most frequently classified as urinary urgency or mild urinary retention (29.55\%).14,17 The frequency of each category of bladder dysfunction is presented in Table 2 .

Results also showed a weak $(\mathrm{r}=0.6)$, while significant $(\mathrm{P}=0,043)$ correlation between disease duration and the presence of urinary dysfunction (Figure 3), observing an increase in the degree of urinary dysfunction over the years.

\section{Outcome of urological complemen-} tary exams

The analysis of complementary urological exams identified abnormalities in $37.7 \%$ of urine tests (chi-squared 0.069 ), in $8.2 \%$ of ultra- sound examinations (chi-squared 0.164), and in $66.7 \%$ of urodynamic evaluations $(\mathrm{P}=0.001)$. A comparison of the results from complementary examinations from asymptomatic patients and those with urinary dysfunction is presented in Table 3. The main abnormality found in the urodynamic exam was an overactive neurogenic bladder, as shown in Table 4.

\section{Discussion}

MS is an immune-mediated disease associated with the Human Leucocitary Antigen (HLA) system. The inflammatory process targets the oligodendrocytes, causing multiple myelin injuries in different topographies of the

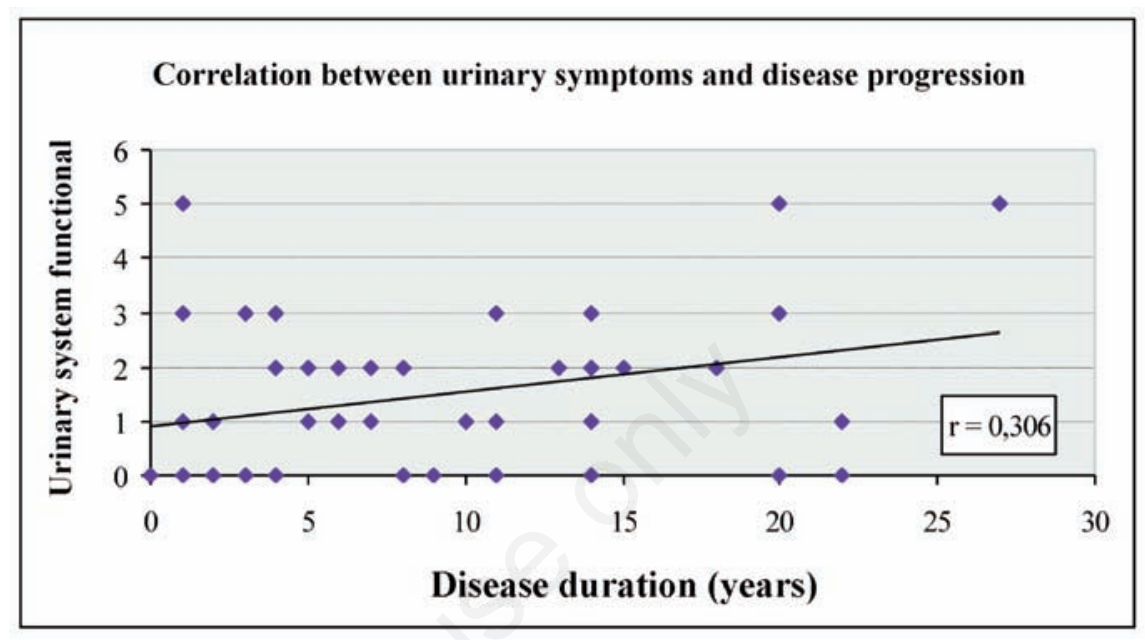

Figure 3. Correlation of disease duration with urinary dysfunction according to Kurtkze's adapted functional classification. P-value (one-sided Mann-Whitney test) $=\mathbf{0 . 0 4 3}$.

Table 2. Kurtzke's adapted classification of Bladder function according to functional systems in multiple sclerosis patients.

\begin{tabular}{llccc} 
Score & Bladder's functional system & $\begin{array}{c}\text { Total of patients } \\
n=61\end{array}$ & $\begin{array}{c}\text { MS-WW } \\
(\mathbf{n}=52)\end{array}$ & $\begin{array}{c}\text { VS-PP } \\
(\mathrm{n}=9)\end{array}$ \\
0 & Normal & $19(31.1 \%)$ & $19(36.5 \%)$ & $0(0 \%)$ \\
1 & Urinary urgency or light urinary retention & $18(29.5 \%)$ & $18(34.6 \%)$ & $0(0 \%)$ \\
\hline 2 & Moderate alteration* & $11(18.0 \%)$ & $8(15.4 \%)$ & $3(33.3 \%)$ \\
3 & Frequent urinary incontinence & $9(14.8 \%)$ & $5(9.6 \%)$ & $4(44.5 \%)$ \\
\hline 4 & Near-frequent bladder catheterization & $0(0 \%)$ & $2(3.9 \%)$ & $0(0 \%)$ \\
5 & Loss of bladder function & $4(6.6 \%)$ & $2(22.2 \%)$ & \\
\hline
\end{tabular}

*Intermittent urethral self-catheterization with manual compression for bladder unload.

Table 3. Results from urological complementary exams in multiple sclerosis female patients.

\begin{tabular}{|c|c|c|c|c|c|c|}
\hline \multirow[t]{3}{*}{ Characteristics } & \multirow{2}{*}{\multicolumn{2}{|c|}{$\begin{array}{c}\text { Total } \\
(n=61)\end{array}$}} & \multicolumn{4}{|c|}{ Urinary abnormalities } \\
\hline & & & \multicolumn{2}{|c|}{ Yes $(n=45)$} & \multicolumn{2}{|c|}{ No $(n=16)$} \\
\hline & n. & $\%$ & n. & $\%$ & n. & $\%$ \\
\hline \multicolumn{7}{|l|}{ Urodynamic test } \\
\hline Normal & 17 & 33.3 & 5 & 13.5 & 12 & 85.7 \\
\hline Abnormal & 34 & 66.7 & 32 & 86.5 & 2 & 14.3 \\
\hline \multicolumn{7}{|c|}{ Urinary tract ultrasound } \\
\hline Normal & 56 & 91.8 & 40 & 88.9 & 16 & 100 \\
\hline Abnormal & 5 & 8.2 & 5 & 11.1 & 0 & 0 \\
\hline \multicolumn{7}{|l|}{ Simple urine test } \\
\hline Normal & 38 & 62.3 & 25 & 55.6 & 13 & 81.3 \\
\hline Abnormal & 23 & 37.7 & 20 & 44.4 & 3 & 18.8 \\
\hline
\end{tabular}


Table 4. Urodynamic evaluation in multiple sclerosis disease female patients $(n=51)$.

\begin{tabular}{lcc} 
Urodlynamic evaluation results & n. & Frequency \\
Normal & 17 & 33.3 \\
Abnormal & 34 & 66.7 \\
\hline Overactive neurogenic bladder & 16 & 31.4 \\
Overactive neurogenic bladder with sphincter & 12 & 23.5 \\
asynchrony or post-voiding residue & & 7.8 \\
\hline Limp bladder, Hipo or No-reflex bladder & 4 & 3.9 \\
Urethral hyper-motility or bladder sphincter incompetence & 2 & 100 \\
\hline Total & 51 &
\end{tabular}

Note: 10 patients without final results

CNS. It may present polymorphic symptoms. Bladder dysfunction is one of the seven most frequent neurological manifestations, along with motor dysfunction, cerebellar, brainstem, sensory, visual and mental impairment. The disease has no biological markers for laboratory identification, so it needs to be differentiated from other diseases that affect the CNS by means of clinical data and complementary examinations, especially MRI and cerebrospinal fluid analysis. Therefore, diagnosis is complex, especially in the early stages.

In this study, we described the urinary symptoms at onset and long-term outcome in patients with MS in a unique series of female patients.

Urinary symptoms were reported by $63.5 \%$ of patients with RRMS and $100 \%$ of patients with PPMS $(\mathrm{P}=0.053)$. This demonstrates the impact of the clinical course of the disease in the presence of urological abnormalities. Although statistical analysis show only a tendency, it is observed that with the evolution time, especially in primary progressive clinical forms, symptoms of bladder or sphincter dysfunction and are always present, usually caused by spinal cord involvement. The prevalence of urinary symptoms was higher than that described in the Brazilian multicenter study, ${ }^{11,14}$ where bowel and bladder sphincter control dysfunctions were reported by $29 \%$ in RRMS ( $\mathrm{n}=467)$, with a mean disease duration of 7.5 years, and by $52.72 \%$ in PPMS ( $\mathrm{n}=55)$, for 8.72 years of disease on average. One possible explanation for the higher prevalence of bladder dysfunction could be the difference in the methodology used for data collection. While the present study was conducted with the contribution of urologists and patients were actively questioned about specific urinary symptoms commonly found in cases of neurogenic bladder, the multicenter study was conducted only by neurologists and urinary symptoms were not actively questioned.

The central control system of the bladder sphincter was the second functional system most affected in MS in early stages. Urinary incontinence, urinary hesitancy, urinary retention, urinary urgency and incomplete bladder emptying were reported by $45 \%$ of patients in their first symptomatic episode. Isolated bladder dysfunction was not reported as a clinically isolated syndrome, but always in association with another neurological dysfunction, particularly motor syndrome. This fact becomes especially important for young patients that seek specialized care complaining of urinary dysfunction. A carefully clinical history should be taken with a focus on other neurological dysfunctions that could raise the hypothesis of MS. 18

It is important to highlight the contribution of the urodynamic evaluation to substantiate the diagnosis of MS. This complementary exam can objectively demonstrate bladder dysfunction in patients with mild symptoms, like urinary hesitancy and urinary urgency, or even in asymptomatic patients. Likewise, $86.5 \%$ of symptomatic patients had abnormal urodynamic results and $14.3 \%$ of the urodynamic studies performed in asymptomatic patients were abnormal $(\mathrm{P}<0.001)$. Koldewijn et al. ${ }^{19}$ confirmed the presence of urinary tract dysfunction by abnormal findings in urodynamic exams in $100 \%$ of MS patients with urinary symptoms and in 52\% of asymptomatic patients in their study. Abnormal urinalysis results were observed in $44.4 \%$ of patients with urinary symptoms, while only $18.8 \%$ of urine tests were abnormal in asymptomatic patients $(\mathrm{P}=0.069)$.

Current episodes of urinary incontinence were reported by $45.9 \%$ of patients, including other milder symptoms of bladder dysfunction, like urinary urgency and nocturia. Carneiro et al. ${ }^{20}$ reported a rate of $15.7 \%$ of female patients aged over 15 complaining of urinary incontinence in the general population, with a frequent association of symptoms like urinary urgency and nocturia.6,16 Our result differs from findings in the general population showing a direct correlation between advanced age and frequency of urinary incontinence in women.6,16 This finding reinforces the impact of MS disease on urinary dysfunction. We also observed that as disease progressed, there was a significant increase in the Urinary Functional Scale $(\mathrm{P}=0.043)$, a comparison never studied by other authors.

The present data derived from urodynamic studies showed abnormal results in 34 patients (66.7\% of tests), with detrusor overactivity being the most frequent finding. Thirty-two of 34 patients with abnormal urodynamic test had urinary complaints and 2 were asymptomatic. In the other way, 5 patients with normal urodynamic test had urinary urgency symptoms (chi square; $\mathrm{P}<0.001)$. These finding reinforces the necessity of urodynamic testing as a clinical routine in patients with MS. This data confirms the results by Betts et al.,12 who found that $47 \%$ of patients with high post-voiding residue had no sensation of incomplete emptying, whereas $83 \%$ of patients with complaints of incomplete emptying had post-voiding residue over $100 \mathrm{~mL}$. Altogether, these facts demonstrate the importance of urodynamics in the follow-up of this specific population. ${ }^{15}$

As for imaging results, $11.1 \%$ patients with urinary symptoms had abnormal ultrasound exams, mostly stones and a few anatomical abnormalities, such as ureteral duplication. In asymptomatic patients, all ultrasound examinations were normal, with no significant difference observed between the groups $(\mathrm{P}=0.164)$.

Interestingly, only 4 (6.6\%) patients had been seen by an urologist throughout the course of the disease. Additional urological examinations had been performed previously in $16(26.2 \%)$ patients, of whom $6.2 \%$ were requested by the urologist, and of these, only one was an urodynamic study; the other abnormal test was the urinalysis. This test is usually requested by a neurologist before the prescription of pulse therapy with high doses of parenteral corticosteroids or immunoglobulin for the treatment of relapses in MS.

\section{Conclusions}

During the study, we observed that the urologist was particularly important for the timely indication of antibiotics in cases of urinary tract infection, medications for neurogenic bladder control and for indication and proper management of intermittent bladder catheterization and long-term indwelling urinary catheterization.

This study showed that there is a clear need for a multidisciplinary approach of MS patients, especially with the cooperation of a urologic medical team. Also, we showed that urinary incontinence was the most common 
symptom followed by urinary urgency, and detrusor hyperactivity was the most frequent abnormality in urodynamic test. In our opinion, urodynamic test reveled to be a good tool for both diagnosis and monitoring of urinary dysfunction in patients with MS.

\section{References}

1. Compston A, Coles A. Multiple sclerosis. Lancet 2002;359:1221-31.

2. Andersson KE. Treatment of overactive bladder: other drugs mechanisms. Urology 2000;55:51-7.

3. Barbalias GA, Liatsikos EN, Passakos C, et al. Urologic manifestations of multiple sclerosis: proposed treatment algorithms. Int Urol Nephrol 2001;32:345-8.

4. Hennessey A, Robertson NP, Swingler R, Compston DA. Urinary, faecal and sexual dysfunction in patients with multiple sclerosis. J Neurol 1999;246:1027-32.

5. Bruschini H, Kano H, Damião R. I Consenso Brasileiro - Incontinência urinária, uroneurológica, disfunções miccionais. 1 ed. São Paulo: Sociedade Brasileira de Urologia; 1999. pp 1-16.

6. Onal B, Silva A, Buldu I, et al. Voiding dysfunction due to muliple sclerosis: a large scale retrospective analysis. Int Braz J Urol 2009;35:326-30.

7. Giannantoni A, Scivoletto G, Di Stasi SM, et al. Urological dysfunctions and upper urinary tract involvement in multiple sclerosis patients. Neurourol Urodyn 1998; 17:89-98.

8. Nitti VW, Combs AJ. Correlation of Valsalva leak point pressure with subjective degree of stress urinary incontinence in women. $\mathrm{J}$ Urol 1996;155:281-5.

9. Giannantoni A, Scivoletto G, Di Stasi SM, et al. Lower urinary tract dysfunction and disability status in patients with multiple sclerosis. Arch Phys Med Rehabil 1999;80: 437-41.

10. Hald T, Bradley WE. Neuroanatomy. In: Hald T, Bradley WE, eds. The urinary bladder neurology and dynamics. Baltimore: Lippincott Williams \& Wilkins; 1982. pp 521.

11. Alvarenga RP, Leon SV, Santos CMM. South Atlantic Project: a Brazilian Multiple Sclerosis Trial. In: Nogales J, Arryada C, eds. Esclerose Multipla - una Mirada íbero latina-americana. led. Santiago: Nogales e Cia; 2002.

12. Callegaro D, Goldbaum M, Morais L, et al. The prevalence of multiple sclerosis in the city of São Paulo, Brazil, 1997. Acta Neurol Scand 2001;104:208-13.

13. Kurtzke JF. Rating neurologic impairment in multiple sclerosis: an expanded disability status scale (EDSS). Neurology 1983;33:1444-52.

14. Papais-Alvarenga RM, Alves-Leon SV, Santos CMM, et al. SIAPEM, a Brazilian and South American database for multiple sclerosis. J Neurol Sci 1997;150:S187.

15. Zorzon M, Zivadinov R, Monti Bragadin L, et al. Sexual dysfunction in multiple sclerosis: a 2-year follow-up study. J Neurol Sci 2001;187:1-5.

16. McDonald WI, Compston A, Edan G, et al. Recommended diagnostic criteria for multiple sclerosis: guidelines from the International Panel on the diagnosis of multiple sclerosis. Ann Neurol 2001;50:121-7.

17. Poser CM. The pathogenesis of multiple sclerosis. Additional considerations. J Neurol Sci 1993;115:S3-15.

18. Janardhan V, Bakshi R. Quality of life in patients with multiple sclerosis: the impact of fatigue and depression. J Neurol Sci 2002;205:51-8.

19. Koldewijn EL, Hommes OR, Lemmens WA, et al. Relationship between lower urinary tract abnormalities and disease-related parameters in multiple sclerosis. J Urol 1995;154:169-73.

20. Carneiro K, Bruschini H, Srougi M, et al. Prevalence of urinary incontinence in unselected Brazilian community-living women over fifteen years old, by direct approach. Abstract no. 240. Proceedings of the International Continence Society, 33rd Annual Meeting. Florence, Italy, 2003. pp. 138-139. 